\title{
Temperature, age of mating and starvation determine the role of maternal effects on sex allocation in the mealybug Planococcus citri
}

\author{
Laura Ross • Elizabeth J. Dealey • Leo W. Beukeboom • \\ David M. Shuker
}

Received: 5 July 2010 /Revised: 11 October 2010 / Accepted: 19 October 2010 /Published online: 10 November 2010

(C) The Author(s) 2010. This article is published with open access at Springerlink.com

\begin{abstract}
Environmental effects on sex allocation are common, yet the evolutionary significance of these effects remains poorly understood. Environmental effects might influence parents, such that their condition directly influences sex allocation by altering the relative benefits of producing sons versus daughters. Alternatively, the environment might influence the offspring themselves, such that the conditions they find themselves in influence their contribution to parental fitness. In both cases, parents might be selected to bias their sex ratio according to the prevailing environmental conditions. Here, we consider sex allocation in the citrus mealybug Planococcus citri, a species with an unusual genetic system in which paternal genes are lost from the germline in males. We test environmental factors that may influence either female condition directly (rearing
\end{abstract}

Communicated by N. Wedell

L. Ross

Theoretical Biology, University of Groningen,

P.O. Box 14, 9750 AA Haren Groningen, The Netherlands

L. Ross $(\bowtie)$

Institute of Evolutionary Biology, School of Biological Sciences,

University of Edinburgh,

West Mains Road,

Edinburgh EH9 3JT, UK

e-mail: 1.ross@rug.nl

E. J. Dealey • D. M. Shuker

School of Biology, University of St Andrews,

Harold Mitchell Building, St Andrews,

Fife KY16 9TH, UK

L. W. Beukeboom

Evolutionary Genetics, Centre for Ecological and Evolutionary

Studies, University of Groningen,

P.O. Box 14, 9750 AA Haren Groningen, The Netherlands temperature and food restriction) or that may be used as cues of the future environment (age at mating). Using cytological techniques to obtain primary sex ratios, we show that high temperature, older age at mating and starvation all affect sex allocation, resulting in femalebiased sex ratios. However, the effect of temperature is rather weak, and food restriction appears to be strongly associated with reduced longevity and a truncation of the usual schedule of male and offspring production across a female's reproductive lifetime. Instead, facultative sex allocation seems most convincingly affected by age at mating, supporting previous work that suggests that social interactions experienced by adult $P$. citri females are used when allocating sex. Our results highlight that, even within one species, different aspects of the environment may have conflicting effects on sex allocation.

Keywords Sex allocation $\cdot$ Sex determination $\cdot$ Maternal condition $\cdot$ Temperature $\cdot$ Ageing

\section{Introduction}

Sex allocation is an important reproductive decision that can have significant effects on an individual's fitness. There is a rich theory-base predicting optimal sex ratios under different conditions, and observed sex allocation patterns often closely match the theoretical predictions, especially in insects (West 2009). The different aspects of sex allocation theory all share conceptual space in terms of fitness returns through sons and daughters, with different "theories" basically dealing with the different ways in which male and female offspring can contribute to parental fitness and how parents and the environment influence this fitness return (Hamilton 1967; Charnov 1982; West 2009). 
Environmental factors acting on parents can affect sex allocation through parental condition: Trivers and Willard (1973) showed that, if there is variation in parental condition and if the fitness of one offspring sex is more strongly affected by their parent's condition, then parents should bias their offspring sex ratio towards the sex that either benefits most or suffers least from their condition. In other words, if the environment affects the condition of a female in such a way that she must invest less in her offspring and one sex suffers less from this reduced investment, a female should bias her sex ratio towards that sex. Several (environmental) factors are known to affect both parental condition and offspring sex ratio. These factors include extreme temperature, drought, parental age and lack of resources (Cockburn et al. 2002; Sabelis et al. 2002; West and Sheldon 2002; Sheldon and West 2004; West 2009).

Alternatively, environmental factors acting upon the offspring themselves can alter sex allocation, as such effects might again influence the fitness return parents get from their offspring (Trivers and Willard 1973). If environmental conditions affect the fitness of male and female offspring differently, and parents can predict the environment their offspring will experience, parents should bias the sex ratio towards the offspring sex with the highest fitness under these conditions. A variety of factors could have differential fitness effects on offspring. One particular factor that has been the focus of many sex allocation studies is the level of competition between kin, as competition between siblings can reduce the fitness return of offspring to their parents, and parents are therefore expected to overproduce the sex that suffers least from kin competition (local resource competition, theory, LRC: Hamilton 1967; Clark 1978; Charnov 1982; West 2009). Data supporting the role of different forms of LRC are abundant, with many of the best examples coming from parasitoid wasps (West et al. 2005; West 2009). Other environmental factors can have similar sex-specific fitness effects, for instance, if the environment influences offspring survival, reproductive success or dispersal ability. These differential effects on offspring fitness have been used to explain environmental effects on sex allocation in a variety of taxa. Examples include the effect of high temperature in the spider mite Tetranychus mcdanieli (Roy et al. 2003), host size and quality in several species of parasitoid wasps (Charnov et al. 1981; Murdoch et al. 1992; West 2009) and female mating age in the mealybug Planococcus citri (Werren and Charnov 1978).

Environmental conditions experienced by parents can therefore influence their sex allocation decisions in two ways: either directly by influencing parental condition, or indirectly by being used as a cue of offspring fitness. In the latter case, parental condition may be completely unaffect- ed, but the environment a parent experiences nevertheless predicts the fitness returns through sons and daughters. Of course, effects at these two levels might interact if parental condition also reflects the offspring environment, making it hard to disentangle the underlying mechanisms (Wild and West 2007). Whilst anecdotal evidence of effects of environmental conditions on sex ratio are numerous, many of these results come from studies focussing on other aspects of life history and experimental studies focussing directly on its effects on sex ratio are rare (Roy et al. 2003). Finally, the underlying mechanisms are often obscured, as in many studies, it is impossible to separate the effects of differential mortality from the effect of adaptive sex ratio adjustment. Here, we present the results of an experimental study on the effect of a variety of environmental conditions on sex allocation in the mealybug $P$. citri.

In $P$. citri, several factors experienced by females have been found to affect sex allocation. These include population density (Varndell and Godfray 1996; Ross et al. 2010a), temperature (James 1937; Nelson-Rees 1960), and age (James 1938; Nelson-Rees 1960; Ross et al. 2010a). The observed effects are of particular interest for two reasons. First, mealybugs have an unusual genetic system, paternal genome elimination (PGE), whereby both sexes develop from fertilised eggs, but, in males, the paternal genome is deactivated during development and lost from the germline during spermatogenesis (Schrader 1921). As a consequence, males have haploid gene expression and only transmit maternal genes (i.e., PGE is akin to true haplodiploidy in terms of transmission genetics and gene expression patterns; Brown and Nelson-Rees 1961; Brown and Nur 1964; Nur 1980). An increased understanding of sex allocation patterns in mealybugs might therefore yield insight into the potential evolutionary advantage of this extraordinary mode of sex determination.

Second, there is strong sexual dimorphism in several morphological and life history traits in mealybugs suggestive of different patterns of selection on males and females (Gullan and Kosztarab 1997). Adult males are winged whilst adult females typically lack wings, have reduced antennae and legs, and exhibit a mostly sedentary habit. In addition, males only feed for the first two instars, whilst females feed throughout their life (Gullan and Kosztarab 1997). These adaptations indicate different resource requirements and dispersive abilities of males and females, such that maternal condition and the environment an offspring finds itself in may differentially influence the fitness returns of sons and daughters (see methods for more details on $P$. citri life history). Due to their dispersive abilities and lesser reliance on larval feeding, males may be less influenced by the environment, but more by maternal condition (if that influences egg size and provisioning). Reduced maternal condition may therefore favour daughter 
production, whilst poorer-quality offspring environments may favour male production. That said, earlier studies showed that both high temperature and ageing of mothers, which we might expect to be related with reduced maternal condition, result in a male-biased sex ratio (Nelson-Rees 1960). More recently, two studies have found effects of density on sex allocation (Varndell and Godfray 1996; Ross et al. 2010a), although the results differed and the cause for this difference could not be identified. Density can influence both maternal quality (if resource competition in high density patches influences maternal condition) and can also be an indicator of the degree of competition that offspring will experience. Previous experiments did not make this distinction between possible effects explicit. However, both studies suggested that resource availability might have a profound effect on sex allocation, and we felt that a renewed attempt to test environmental effects on sex allocation was necessary in order to understand sex allocation patterns in P. citri.

We performed two experiments that manipulated three environmental factors that differed in whether they directly influenced maternal condition (rearing temperature and food deprivation) or whether they might signal aspects of the environment (availability of males, manipulated as age at first mating, and representing a measure of population density). In the first experiment, we manipulated temperature and age at first mating in a fully factorial experiment. In this experiment, we used two temperature treatments: $25^{\circ} \mathrm{C}$, which is the temperature at which the stock cultures are kept and $30^{\circ} \mathrm{C}$, as previous experiments have shown that females kept at this temperature have a reduced life span and reproductive success. In our second experiment, we tested the role of food deprivation on sex allocation. If maternal condition influences sex allocation in P. citri, we predict sex ratio changes associated with rearing temperature and food deprivation. As male offspring are thought to depend more on maternal investment than female offspring (which feed throughout their lives), we predict that maternal stress is associated with a greater production of female offspring. If sex allocation is more strongly influenced by cues about the offspring environment, delayed mating (due to low availability of males) might signal low density, and thus we predict the greater production of female offspring (Ross et al. 2010a).

\section{Methods}

Study organism

The citrus mealybug, P. citri (Pseudococcidae: Coccoidea: Hemiptera) is a cosmopolitan sap-feeding plant pest species (Gullan and Kosztarab 1997). It is sexually dimorphic, with the males being small and wingless whilst the females are larger, sedentary and covered in wax (see Fig. 1 for an illustration of both sexes). The adult males become sexually mature at approximately 29 days (when reared at $25^{\circ} \mathrm{C}$ ) and have a short life span of approximately 2 days, during which they do not feed. The females become sexually mature at approximately the same time, and have been reported to survive up to 120 days under laboratory conditions (Nelson-Rees 1960). Females can lay several hundred eggs during their lifetime, and these eggs are laid in a fibrous ovisac located under and behind her body. The larvae ('crawlers') hatch about 2-3 days after egg-laying, and the first instar larvae are highly mobile (Gullan and Kosztarab 1997), although generally crawlers settle closely to where they were born. The two sexes become distinguishable in the late second instar at approximately 1620 days post-oviposition when the males pupate (Ross et al., submitted). The laboratory stock culture of $P$. citri that was used for the experiments was obtained from Wye College, University of London, and originally collected from a nearby glasshouse, and has been maintained through mass cultures on potato (cultivar Desiree) at $25^{\circ} \mathrm{C}$ and 60 $70 \%$ humidity, under a 12:12 light/dark cycle.

\section{Experiment 1: temperature and age at mating}

The first experiment consisted of two rearing temperatures and two mating ages in a fully factorial design. The two rearing temperatures were $25^{\circ} \mathrm{C}$ and $30^{\circ} \mathrm{C}$, and the two mating ages were 29 days and 63 days (see Fig. 1). The whole experiment was repeated twice, resulting in two blocks. The earlier mating age was chosen as females become sexually mature at approximately 29 days; the older age of 63 days was selected following the data from Nelson-Rees (1960), which shows that the sharpest rate of increase in proportion of males occurs after 50 days. Effects of mating age and temperature on males were controlled for by mating all experimental females with 29 -day-old males which had been raised at $25^{\circ} \mathrm{C}$.

The females used in all treatments were raised at $25^{\circ} \mathrm{C}$ till they became detectably female (day 20 ), then females were isolated and transferred to either the $25^{\circ} \mathrm{C}$ or $30^{\circ} \mathrm{C}$ treatment group. Females were mated and remained at these temperatures until they finished ovipositing. All mealybugs used in the experiment were obtained by allowing 50 randomly selected females to oviposit overnight. The egg masses from these females were transferred to fresh potatoes in order to obtain the stock used for the experiments, ensuring constant age for all individuals used.

At day 20, when the sexes of the crawlers became distinguishable, females were randomly selected from the experimental stock and each individual female was assigned to a box in one of the four treatment groups, so that each box 


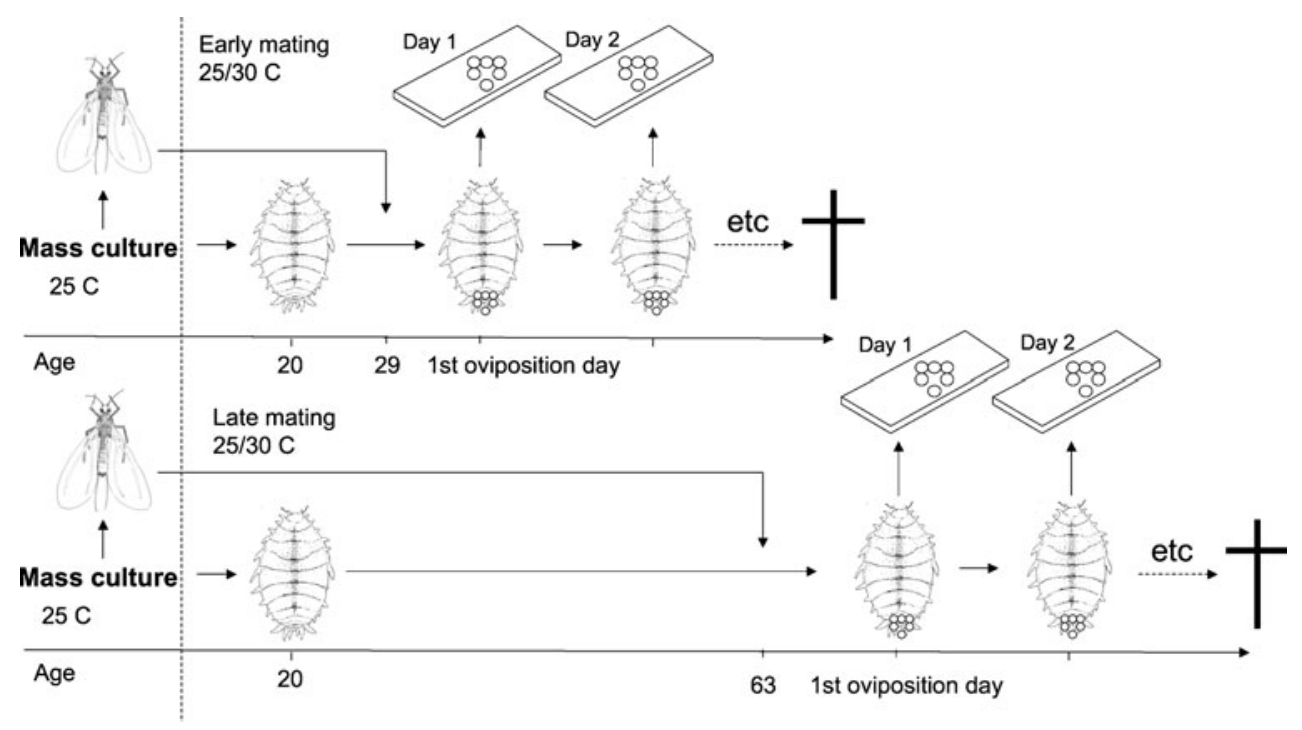

Fig. 1 A schematic representation of experiment 1 manipulating temperature and mating age. The axis shows the age of the females. The top panel shows the timing of events - when females are isolated from the mass culture, when males are introduced and when females started ovipositing and eggs were collected - for the early mating

contained one solitary female. Treatment boxes (plastic boxes with a volume of 1.11 , covered with fine mesh) were set up as follows: a single freshly sprouted potato was randomly selected, the weight recorded and then placed in the box on a single sheet of paper towel. The box was then randomly assigned to a treatment, and 30 boxes were set up for each treatment group. The treatment boxes were then placed at either $25^{\circ} \mathrm{C}$ or $30^{\circ} \mathrm{C}$, both at $60-70 \%$ humidity and under a 12:12 light/dark cycle. In addition, males were isolated from the experimental stock and placed into individual glass tubes, which were then maintained at $25^{\circ} \mathrm{C}, 60-70 \%$ humidity and under a 12:12 light/dark cycle. Both sexes were then allowed to develop for a further 9 days. Additionally, at day 34, a further 50 ovipositing females were randomly selected from the stock cultures and given fresh sprouting potatoes, as described before, in order to generate males for the late mating treatments. The egg masses from these females were again removed after 1 day and placed on fresh potatoes.

For the early mating treatments at day 29 , two males were randomly selected and released into each of the boxes in the early mating treatments (two males were used to ensure that all females were inseminated). For the late mating treatments, males were introduced into the female boxes at day 63 (Fig. 1). All females were checked daily, from the day after mating for signs of oviposition. As soon as the females began oviposition, the egg masses were collected daily until the female died (Fig. 1). Egg masses were fixed and stored prior to sexing (see below for fixation and staining methods).

In total, 95 females successfully started oviposition and were included in the analysis. Mortality before the start of treatments, whilst the bottom panel shows the late mating treatments. Temperatures during each of the experimental treatments are indicated in the figure: $25^{\circ} \mathrm{C}$ in the mass culture and during the rearing of males and either $25^{\circ} \mathrm{C}$ or $30^{\circ} \mathrm{C}$ during the experimental stage, depending on the temperature treatment in both the early and late mating treatments

oviposition was high for the late mating treatments, especially those kept at $30^{\circ} \mathrm{C}$. Additionally, in the late mating treatments, several females failed to start ovipositing. In a few cases, females in the late mating treatment started ovipositing before males were introduced; as $P$. citri reproduction is strictly sexual (Borges da Silva et al. 2010), this was probably due to males managing to enter the treatment boxes. These females were excluded from the experiment.

\section{Experiment 2: food restriction}

To test the effect of food restriction, we either removed mated females from their food source (food-restricted treatment) or moved mated females to a new food source (control). For the control females, we also noted daily if females were able to feed (present on the potato); since mealybug females are almost completely sedentary, this was assumed to provide reasonable information on their opportunity to feed.

In order to obtain individuals of known age that could be used for the experiment, we randomly took 20 egg-laying females from the mass culture and provided them with fresh potatoes. The females were allowed to lay eggs for $24 \mathrm{~h}$. The eggs were allowed to hatch and the crawlers were raised until 19 days after they were laid. By this time, males had pupated and were easily recognisable, and males and females were isolated in order to avoid early mating: females were removed from the mass culture and each individually placed on a new potato in a smaller box. Males were isolated in small glass tubes. After 29 days, two adult males were introduced into each box again to make sure 
that all females would be inseminated. The next day (day 30 ), we removed the males, and the females were randomly assigned to one of the two treatments: food restriction or the control. In the food restriction treatment, females were removed from the potato and placed back in their box on a piece of paper towel, whilst the control treatment females are removed from the potato and placed back in their box on a new potato. Subsequently, females were checked each day for signs of egg-laying, and eggs were removed and fixed. Additionally, for the control females, we also noted whether individuals were sitting on the potato or on the paper towel. In total, 50 out of the 56 females in the experiment started oviposition, with 11,189 eggs being counted and $98.5 \%$ of the eggs successfully sexed. Eggs were counted on the first and every alternating day until the females died.

\section{Fixation and staining}

Immediately following collection, we placed a few drops of fixative solution (Carnoy's fluid: four parts chloroform/ three parts ethanol/one part glacial acetic acid) on the egg mass and removed the protecting fibres using a small rod under a dissecting microscope. We then transferred the eggs to an Eppendorf tube containing the fixative solution and stored them in a fridge at $4^{\circ} \mathrm{C}$ for at least $24 \mathrm{~h}$ and up to a maximum of 3 days. At the end of this period, the eggs were transferred into $90 \%$ ethanol solution to prevent DNA degradation and stored at $4{ }^{\circ} \mathrm{C}$.

To determine the sex of each embryo, we transferred eggs from the ethanol solution to a glass slide, stained them with DAPI (Sigma D9564, diluted 1:1,000 in PBS) and then sealed a cover slip over the stained embryos. We then examined the eggs under a fluorescence microscope at $\times 200$ magnification and determined the sex of each embryo. The males can be differentiated from the females as their condensed paternal genome stains heavily and forms a brightly coloured body in the nuclei of male cells (Ross et al. 2010a). The sex of each embryo (female, male, unknown) was recorded for every clutch (experiment 1 ) or every other clutch (experiment 2) from all females in the treatments. For experiment 1, we sexed 28,086 , with a $98 \%$ success rate, whilst for experiment 2, we sexed 11,189 embryos with a $98.5 \%$ success rate.

\section{Data analysis}

All data analyses were performed using the statistical programme R (R Development Core Team 2009). For generalised linear mixed models, the $\mathrm{R}$ package nlme was used (Pinheiro et al. 2007). The relationships between the life history traits-fecundity, longevity and ovipositionwere explored using generalised linear models with
Gaussian error structures with a log transformation or with quasi-Poisson error structures in the case of clutch size, and we consider these factors as measures of maternal condition. The lifetime sex ratios were analysed using generalised linear models with quasi-binomial error structure to correct for overdispersion. The sex ratio data per day were analysed by using a generalised linear mixed model approach with female identity fitted as a random effect and with arcsin square root transformed sex ratios, assuming a Gaussian error structure. We also used a correction for autocorrelation (corCAR1 function in nlme: Pinheiro et al. 2007). For the analysis of the effects of food restriction on sex ratio, in addition to the treatment for the control females, we also considered female feeding location (on or off the potato). For the analysis per female, we used fitted fraction days feeding (number of days on potato/ length oviposition), whilst for the analysis per day, we fitted the feeding position of the female for each day. These factors were both fitted for control females only, using the "at.level" function in the R package MCMCglmm (Hadfield 2010). Throughout, we consider sex ratio as the fraction of offspring that are male.

\section{Results}

Experiment 1: temperature and mating age

\section{Effects on maternal condition}

The rearing temperature of female mealybugs significantly affected aspects of development, but it was not clear that maternal condition was adversely affected by high temperature. Females reared at the higher temperature lived a

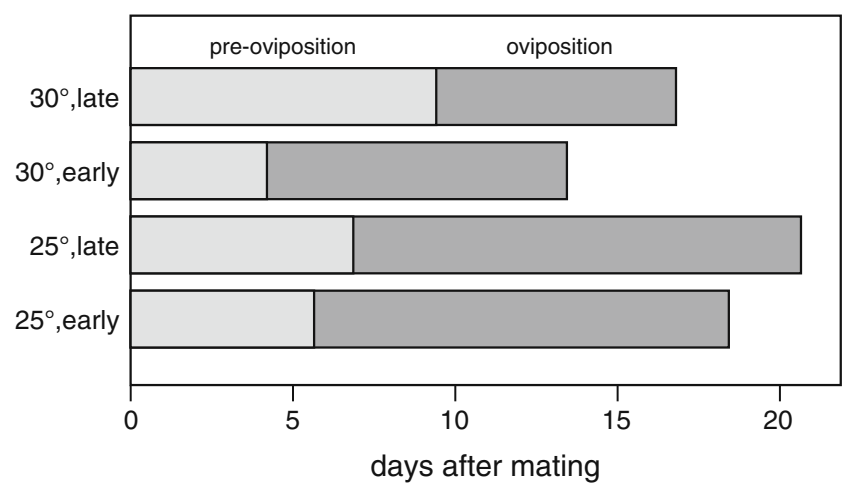

Fig. 2 Mean time between mating and oviposition (light grey bars, "pre-oviposition") and the length of oviposition (dark grey bars, "oviposition") for each treatment in experiment 1 . Times are presented as time after mating (day 29 for the early mating treatments and day 63 for the late mating treatments). Treatments shown from top to bottom: high temperature and delayed mating (" 30 , late"), high temperature and early mating (" 30 , early), low temperature and delayed mating (" 25 , late"), and low temperature and early mating ("25, early") 
shorter span and had a shorter oviposition period $\left(\mathrm{F}_{1,90}=\right.$ 16.86, $p<0.001$; Fig. 2), but they did not produce fewer offspring (mean number of offspring $25^{\circ} \mathrm{C}, 357.6, \mathrm{SE}=$ $\left.34.0 ; 30^{\circ} \mathrm{C}, 348.5, \mathrm{SE}=32.2 ; \mathrm{F}_{1,90}=0.93, p=0.34\right)$, and they did not take significantly longer to commence oviposition $\left(\mathrm{F}_{1,90}=3.45, p=0.07\right)$. There was a significant interaction with age at mating, however (see below).

Age of mating was also associated with aspects of maternal reproductive behaviour and life history. Mating age was strongly associated with female lifespan, consistent with there being a cost of reproduction. Some $79 \%$ of females in the late mating treatment survived until day 63 , when the males were introduced. By this time, only three out of 58 of the early mating females were still alive. However, females from the early and late mating treatments did not differ in the length of their oviposition period post-insemination $\left(\mathrm{F}_{1,89}=0.008, p=\right.$ 0.93). Age at mating was also associated with female egg production, with females producing fewer offspring when mated later in life $\left(\mathrm{F}_{1,89}=15.86, p<0.01\right)$, with the number of offspring even more reduced for the late mating females kept at $30^{\circ} \mathrm{C}$ (Temperature $\times$ Mating time, $\mathrm{F}_{1,87}=13.59, p<0.01$ ). Finally, females in the early mating treatment reared at $30^{\circ} \mathrm{C}$ started ovipositing following insemination earlier than those raised at $25^{\circ} \mathrm{C}$, although females in the late mating treatment took longer to start ovipositing at higher temperature (Temperature $\times$ Age at mating, $\mathrm{F}_{1,87}=17.1, p<0.001$, time post-insemination; Fig. 2). Potato weight did not affect any of these maternal condition factors (all $p>0.2$ ). In summary, temperature appeared to have at most small effects on maternal condition (e.g., no loss of fecundity), whilst females that mated later in life produced fewer offspring, presumably due to that delay.

\section{Sex ratio}

Considering the overall sex allocation across a female's life (see Fig. 3), there was no effect of rearing temperature on sex ratio $\left(\mathrm{F}_{1,93}=1.12, p=0.293\right)$ and a non-significant interaction between temperature and age at mating $\left(\mathrm{F}_{1,91}=\right.$ $3.51, p=0.064)$. There was a significant effect of time of mating on lifetime sex ratio, with females that mated later in life producing a more female-biased sex ratio $\left(\mathrm{F}_{1,92}=\right.$ $6.35, p=0.014)$.

We also analysed the sex ratio per day, and the results are shown in Table 1 and Fig. 4. The number of days since the commencement of oviposition ("oviposition day") was strongly associated with sex ratio (Table 1) and the effect was linear (the quadratic term was non-significant, although there was a significant difference between the quadratic terms of the two temperature treatments: Table 1). In this analysis, temperature was again not significantly associated with sex ratio as a main effect, although both mating time and the interaction between mating time and temperature

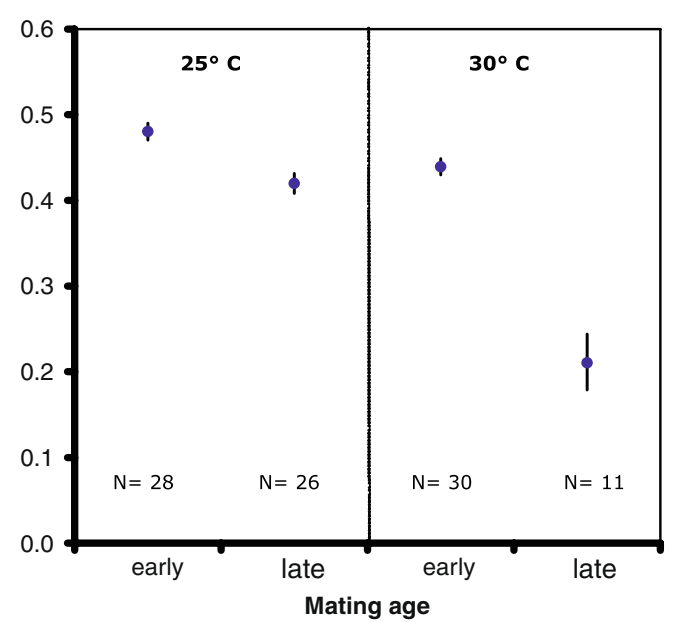

Fig. 3 Mean lifetime sex ratio for each of the four treatment combinations of experiment 1. Results of the low temperature treatments are shown in the left panel of the graph whilst the results of the high temperature treatments are shown in the right panel. The age at which females were mated (early and late) is shown on the $x$ axis. Sex ratios are the proportion of offspring that are male. Error bars show the binomial standard errors

were significant and all of these factors resulted in a more female-biased sex ratio. The results also show that the observed effects on the total sex ratio cannot be fully explained by the different length of the oviposition period between the treatments. Figure 4a shows the pattern of sex ratio production at $25^{\circ} \mathrm{C}$ with females mating at reproductive maturity, with males predominating early on, followed by a female bias, and then a tendency to produce more males again (see also Ross et al. 2010a for discussion of this "standard" pattern). Figure $4 \mathrm{~b}-\mathrm{d}$ shows the sex ratio patterns for the other three treatments. The most notable difference to Fig. 4a is that the initial peak of male bias is less obvious or absent and that there is also no second peak of male production. As such, age of mating and to some extent, temperature, influence the ontogeny of sex allocation across much of the oviposition period, such that we see fewer males produced earlier and fewer males produced later as compared with "standard" lab conditions, generating a more female-biased sex ratio overall.

\section{Experiment 2: food restriction}

\section{Effects on maternal condition}

Food restriction significantly affected maternal condition in several ways, suggesting that food restriction did harm female condition. First of all, females that were starved lived significantly shorter than the control females $\left(\mathrm{F}_{1,48}=\right.$ $12.97, p<0.001)$. Food restriction also affected the number of eggs produced (control females, 276.63, $\mathrm{SE}=26.03$; starved females, 175.00, $\mathrm{SE}=12.92 ; \mathrm{F}_{1,48}=12.17, p=$ $0.001)$. For the control females, the fraction of days spent 
Table 1 ANOVA table showing the results of experiment 1 of temperature and mating age

GLMM with arcsin square root transformed sex ratio, female ID fitted as a random effect and Gaussian errors corrected for autocorrelation

\begin{tabular}{|c|c|c|c|c|}
\hline & numDF & denDF & $F$-value & $p$-value \\
\hline (Intercept) & 1 & 823 & 582.33 & $<0.0001$ \\
\hline Temperature & 1 & 87 & 2.43 & 0.123 \\
\hline Mating age & 1 & 87 & 23.85 & $<0.0001$ \\
\hline Laying day & 1 & 823 & 87.42 & $<0.0001$ \\
\hline Laying day ${ }^{2}$ & 1 & 823 & 0.74 & 0.390 \\
\hline Potato weight & 1 & 823 & 1.46 & 0.228 \\
\hline Block & 1 & 87 & 2.53 & 0.116 \\
\hline Temperature: Mating age & 1 & 87 & 5.22 & 0.025 \\
\hline Temperature: Laying day & 1 & 823 & 0.00 & 0.955 \\
\hline Temperature: Laying day ${ }^{2}$ & 1 & 823 & 4.48 & 0.035 \\
\hline Mating age: Laying day & 1 & 823 & 2.26 & 0.133 \\
\hline Mating age: Laying day ${ }^{2}$ & 1 & 823 & 0.94 & 0.334 \\
\hline
\end{tabular}

on the potato was significantly positively associated with the number of offspring produced $\left(\mathrm{F}_{1,47}=8.60, p=0.005\right)$. Food-restricted females started laying eggs significantly earlier than the control females (mean number of days after mating for control females 4.42, $\mathrm{SE}=0.36$; for starved females 3.00, $\mathrm{SE}=0.10 ; \mathrm{F}_{1,48}=7.46, p=0.009$ ). Among the food-restricted females, those that started laying earlier laid more eggs over their lifetime $\left(\mathrm{F}_{1,24}=6.51, p=0.018\right)$.

\section{Sex ratio}

We again analysed sex ratio in two different ways. First of all, we tested the effect of starvation on the overall sex ratio produced by each female, also fitting the proportion of days control females were observed on the potato (number of days feeding divided by the length of the oviposition period) to control for their feeding behaviour. Food restriction significantly influenced sex ratio, with foodrestricted (i.e. poor condition) females producing more female-biased sex ratios (starvation 0.47, $\mathrm{SE}=0.017$; control 0.53, $\mathrm{SE}=0.018, \mathrm{~F}_{1,48}=7.04, p=0.011$ ). However, the fraction of days control females spent on the potato did not have a significant effect on the sex ratio those females produced $\left(\mathrm{F}_{1,47}=0.78, p=0.382\right)$.

We also analysed the sex ratio per day using a GLMM with female identity as a random effect. For the control
Fig. 4 Mean sex ratio per day with respect to oviposition day (days since the start of oviposition) for each of the four treatment combinations of experiment 1: a Low temperature and early mating; b Low temperature and delayed mating; c High temperature and early mating; d High temperature and delayed mating. Error bars show the binomial standard errors (a)

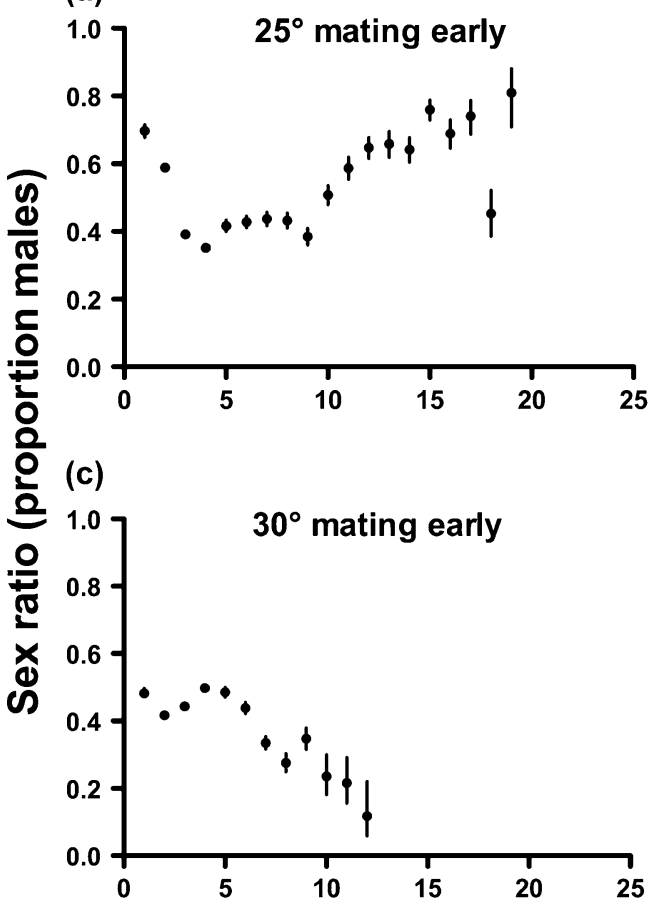

(b)

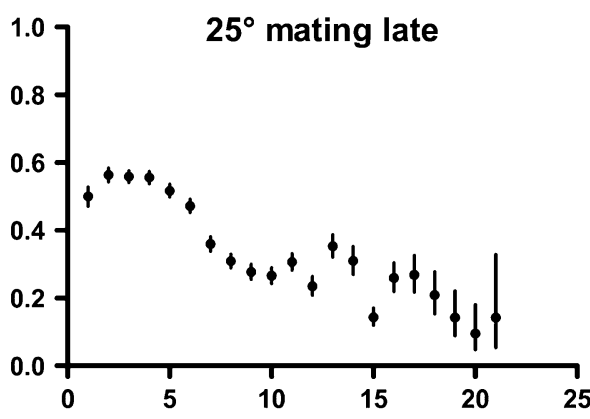

(d)

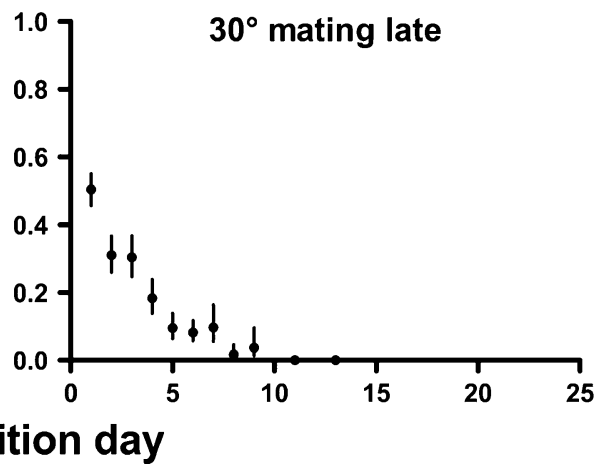


females, we again fitted whether they were on the potato or not on each day. The results are shown in Table 2. First of all, oviposition day had a significant non-linear effect on the sex ratio (Table 2, Fig. 5). Food-restricted females produced a more female-biased sex ratio than the control, with food-restricted females clearly missing the peak in male production late in life (Fig. 5). The feeding opportunities of control females were not associated with sex ratio $\left(t_{217}=0.89, p=0.375\right)$. As both the analysis considering the total sex ratio as the one looking at daily sex ratio patterns show a significant effect of food restriction, it is clear that although some of the effect of the total sex ratio might be influenced by differences in lifespan between the treatments (see Fig. 5); this does not explain the whole effect.

\section{Discussion}

In this paper, we have considered how aspects of the maternal environment influence sex allocation in the mealybug $P$. citri. Our clearest result is that food deprivation reduces maternal condition and leads to a greater production of female offspring. This result suggests that environmental impacts on adult female mealybugs can influence sex allocation and in the predicted direction, if males are more reliant on maternal resources than females. However, the extent to which this sex ratio shift is facultative is perhaps questionable in that the reduced lifespan of these starved females appears to have merely truncated the schedule of production of male and female offspring across the oviposition period, rather than fundamentally changed the rate at which males and females are

Table 2 Contrast table showing the results of experiment 2 of food restriction (starved vs. fed)

\begin{tabular}{lrcccr}
\hline & Value & Std.Error & DF & $t$-value & $p$-value \\
\hline Starved & $\mathbf{0 . 3 5}$ & $\mathbf{0 . 0 5 2}$ & $\mathbf{2 1 5}$ & $\mathbf{6 . 7 7}$ & $<\mathbf{0 . 0 0 0 1}$ \\
Day: Fed & $\mathbf{- 0 . 0 6}$ & $\mathbf{0 . 0 0 8}$ & $\mathbf{2 1 5}$ & $\mathbf{- 8 . 0 9}$ & $<\mathbf{0 . 0 0 0 1}$ \\
Day $^{2}$ : Fed & $\mathbf{0 . 0 1}$ & $\mathbf{0 . 0 0 1}$ & $\mathbf{2 1 5}$ & $\mathbf{8 . 8 1}$ & $<\mathbf{0 . 0 0 0 1}$ \\
Day: Starved & 0.02 & 0.015 & 215 & 1.4 & 0.1627 \\
Day $^{2}$ : Starved & 0.02 & 0.003 & 215 & 5.25 & $<0.0001$ \\
Fed: off potato & 0.21 & 0.08 & 215 & 2.62 & 0.0095 \\
Fed: on potato & 0.30 & 0.075 & 215 & 4.05 & 0.0001 \\
\hline
\end{tabular}

GLMM with arcsin square root transformed sex ratio and Gaussian errors, corrected for autocorrelation. Table shows the (arcsin square root) transformed estimates (in bold) for the first factor level and the difference between the first and remaining factor levels (non-bold), e.g. "Fed: off potato", gives the difference between estimate for females that where starved ("Starved") and those that were not starved, but not feeding. The $p$-value shows the significance of this difference. Likewise "Day: Starved" shows the difference in slope between starved females and control females ("Day: Fed")

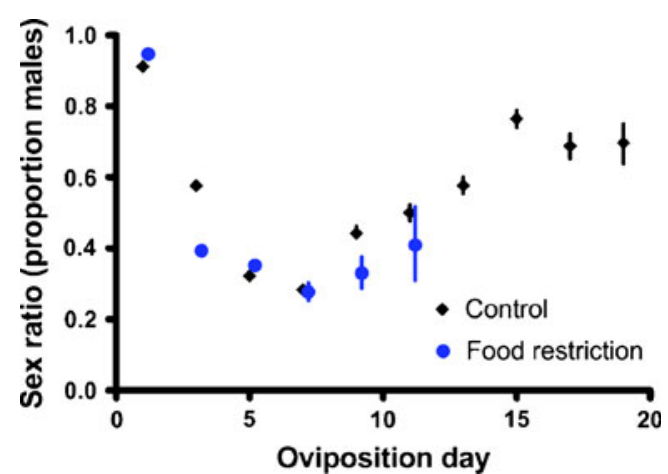

Fig. 5 Mean sex ratio for each day plotted against oviposition day from experiment 2 . The data presented are the number of days since the start of oviposition for the control females (black triangles) and for those that were food-restricted (blue circles)

produced (Fig. 5). The shift in sex ratio across a female's lifespan may then just be an artefact of curtailing the usual pattern of allocation, rather than a deliberate shift in sex ratio strategy.

The other factor that we manipulated and expected to influence maternal condition-rearing temperatureseemed to have a rather negligible effect on maternal condition, at least in terms of egg production (perhaps the closest measure we have to overall fitness). Likewise, temperature was only weakly associated with changes in sex allocation, only significant when interacting with the other factor we manipulated, namely age at mating. This questions the relevance of rearing temperature for sex allocation in P. citri (see below). On the other hand, our environmental factor associated with mate availability - age at mating - although inevitably associated to some extent with female condition in terms of age (but again see below), was indeed associated with changes in sex allocation. Females that experienced delayed mating were more likely to produce females, and this result suggests that the frequency of interactions with males influences sex allocation, which can be interpreted in two ways. First, low encounter rate between adults can signal low population density, which has been shown to be associated with more female-biased sex ratios before in $P$. citri, as observed here (Ross et al. 2010a). Secondly however, Werren and Charnov (1978) have previously interpreted data from $P$. citri that showed increased male production by females that had experienced delayed mating (Nelson-Rees 1960) as proof for an adaptive response by mothers to a shortage of males (invoking classic Düsing-Fisher frequency dependence), but clearly, our data question the generality of that finding, at least in terms of the overall link between mate encounter rate and sex ratio.

The two experiments presented here, combined with our previous work (Ross et al. 2010a), show that female age is associated with sex allocation, in as much as sex ratio 
varies across a female's oviposition period. If we assume that older females are in poorer condition due to the costs of reproduction, then we see that older females under "good" environmental conditions (mated promptly, reared at $25^{\circ} \mathrm{C}$ and not food-restricted) tend to produce more male offspring at the end of their oviposition periods (Figs. 3 and 4, and see Ross et al. 2010a). The key question is whether this later phase of male production signifies, or is a result of, genuine "poorer" female condition? Relevant here are our age at mating treatments in experiment 1. By delaying mating, we delayed oviposition by in excess of 30 days, and yet the initial patterns of sex allocation were generally similar to (or more female-biased than) the beginning of the oviposition period for "standard" females. Thus, age per se does not appear to be associated with sex allocation pattern; rather, it is when during the oviposition period that eggs are laid that is more crucial. Whilst one is not strictly comparing like with like (older, but unmated females will presumably have less resources available for reproduction when they finally commence oviposition), it is striking that Fig. 4b, d are more similar to the first half of the oviposition period of Fig. 4a than the latter half. As such, female age, in itself, is perhaps less of an indicator of female quality; rather, female age appears to interact with other factors such as age at mating and stage of the oviposition period.

As, alluded to above, the results concerning rearing temperature and age at mating found in experiment 1 differ from those observed in earlier studies of sex allocation in $P$. citri (James 1937; James 1938; Nelson-Rees 1960). This could, in part, be due to the fact that our experiment used a different strain than the one that was used in previous studies. However, another explanation is that, in previous experiments, brood sex ratios were determined at the beginning of the third instar when the sexes become distinguishable, whilst we determined the sex of embryos. This means that the difference might be explained by strong differential mortality between the sexes, caused by temperature (experienced by the mother) or mating age. If this is true, we would expect the previous experiments to find a strong reduction in the number of surviving offspring between the treatments: this was indeed the case. For instance, when comparing our results with those obtained by Nelson-Rees (1960), our study shows less of a reduction in clutch size as a result of high temperature and age at mating (see Table 3). This suggests that at least some of the differences between our studies might be explained by differential mortality between the sexes and that both the temperature a female has been raised at and her age at mating affect the mortality of male and female offspring differently. It is important to note that the suggested differential mortality effect of temperature is not the effect of temperature per se but of the temperature experienced by the mother, as in NelsonRees' experiments where all clutches were raised at the same temperature. Thus, daughters from mothers reared at higher temperatures may suffer from higher mortality than their male siblings. The reason for this is unclear. However, it has recently been observed that the titre of the obligate endosymbionts that mealybugs need to survive are affected by host age and temperature, with a strong reduction in the number of endosymbionts in older females (Kono et al. 2008) and in females raised at high temperatures (Buchner 1965). Since it has been suggested that males might be less dependent on the endosymbionts than females (Ross et al. 2010b), the differential survival effects might be explained by the number of endosymbionts a mother is able to transmit to her offspring.

The results from our experiments and earlier studies (Nelson-Rees 1960; Varndell and Godfray 1996; Ross et al. 2010a) show that there is a strong effect of oviposition day on sex ratio, and whilst the evolutionary significance of this pattern is currently unclear (and will be discussed elsewhere, Ross et al., submitted), it is important to consider as it might affect the interpretation of sex allocation data. For example, in our experiment, food restriction seems to give an adaptive pattern if overall sex ratio across a female's whole oviposition period is considered (assuming daughters favoured if mothers are in poor condition), whereas in fact it may be an artefact of a reduced lifespan interfering with the "usual" schedule of sex allocation. If this is right (and
Table 3 Comparison between clutch size effects of different environmental factors between our study and those obtained by Nelson-Rees (1960)

\begin{tabular}{lllll}
\hline Nelson-Rees 1960 & & & This study \\
Treatment & Results & & Treatment & Results \\
\hline Temperature & Clutch size & & Temperature & Clutch size \\
$20-26 \mathrm{C}$ & 527 & $25 \mathrm{C}$ & $357.6( \pm 34.0 \mathrm{SE})$ \\
$29.1-30.1 \mathrm{C}$ & 164.91 & $30 \mathrm{C}$ & $348.5( \pm 32.2 \mathrm{SE})$ \\
Mating age & Clutch size & & Mating age & Clutch size \\
At maturation & 420 & At maturation & $357.6( \pm 34.0 \mathrm{SE})$ \\
70 days & 220 & 63 days & $268.0( \pm 43.5 \mathrm{SE})$ \\
\hline
\end{tabular}


we can accept that it may not be), then adaptive facultative sex allocation with respect to food restriction is questionable. Order effects in sex allocation, where sex ratios change during the oviposition period, are common in other taxa (Hardy 1992), and our results highlight that care needs to be taken when interpreting sex ratios, especially if longitudinal patterns of sex allocation (but within individuals) are not adequately considered.

Our results do support the findings of earlier experiments (Varndell and Godfray 1996; Ross et al. 2010a) showing that female $P$. citri facultatively adjust their sex allocation depending on local conditions (aspects of population density). The mechanism by which they might do this is currently unclear though. Sex chromosomes in mealybugs are absent, and it seems unlikely that autosomal genetic factors determine sex. Recently, several histone proteins involved in the heterochromatization of paternal chromosomes in males have been shown to be present at higher concentrations in male embryos in $P$. citri (Bongiorni et al. 2001; Bongiorni and Prantera 2003; Bongiorni et al. 2004; Bongiorni et al. 2007). It has therefore been suggested that these proteins are also involved in sex determination (Buglia and Ferraro 2004; Ross et al. 2010b), and females might alter the concentration of these proteins in their eggs to change the sex ratio of their broods. Along these lines, Buglia et al.(2009) showed higher concentrations of the histone protein HP1 in eggs of females that were aged prior to mating. However, in that study, they assumed that these females produce male-biased clutches (although the sex ratio data were not provided), whilst in our study we observe the opposite effect of maternal ageing prior to mating. It will be of great interest to test the effect of temperature and starvation on the concentration of HP1 in embryos and to directly link this to sex ratio data, as this will not only help to unravel the sex determination mechanism, but also how females are able to adjust their sex ratio according to environmental cues.

To conclude, our results show that even within a single species different components of the environment can influence sex allocation in complicated and apparently conflicting ways (for instance, if one assumes that certain factors will always influence maternal condition in a negative way). As such, our work reinforces the emerging consensus that multiple forces of sex ratio selection (LRC, Trivers and Willard 1973 and so forth) may act simultaneously in populations (e.g. Pen and Weissing 2002; Wild and West 2007). Whilst this consensus may be intellectually refreshing, it will make determining patterns of causation difficult. Experimental approaches such as those undertaken here are a necessity, as will be a greater understanding of the mechanistic basis of how and when females allocate sex to their offspring (West 2009). The more organisms we determine these mechanisms for the better.
Acknowledgements We are extremely grateful to Mike Copland, not only for kindly providing the cultures used in this study, but also for his invaluable advice on rearing mealybugs. Jarrod Hadfield provided advice on the statistical procedures and helped in the lab. We were supported by the Natural Environment Research Council, the University of Groningen and the University of Edinburgh Development Trust.

Ethical standards The authors declare that the experiments comply with the current laws of the UK, where they were performed.

Conflict of interest The authors declare that they have no conflict of interest.

Open Access This article is distributed under the terms of the Creative Commons Attribution Noncommercial License which permits any noncommercial use, distribution, and reproduction in any medium, provided the original author(s) and source are credited.

\section{References}

Bongiorni S, Prantera G (2003) Imprinted facultative heterochromatization in mealybugs. Genetica 117:271-279

Bongiorni S, Mazzuoli M, Masci S, Prantera G (2001) Facultative heterochromatization in parahaploid male mealybugs: involvement of a heterochromatin-associated protein. Development 128:3809-3817

Bongiorni S, Fiorenzo P, Pippoletti D, Prantera G (2004) Inverted meiosis and meiotic drive in mealybugs. Chromosoma 112:331-341

Bongiorni S, Pasqualini B, Taranta M, Singh P, Prantera G (2007) Epigenetic regulation of facultative heterochromatinisation in Planococcus citri via the Me(3)K9H3-HP1-Me(3)K20H4 pathway. J Cell Sci 120:1072-1080

Borges da Silva E, Mendel Z, Franco J (2010) Can facultative parthenogenesis occur in biparental mealybug species? Phytoparasitica 38:19-21

Brown SW, Nelson-Rees WA (1961) Radiation analysis of a Lecanoid genetic system. Genetics 46:983-1006

Brown SW, Nur U (1964) Heterochromatic chromosomes in Coccids. Science 145:130-136

Buchner P (1965) Endosymbiosis of animals with plant microorganisms. Interscience Publisher, New York

Buglia G, Ferraro M (2004) Germline cyst development and imprinting in male mealybug Planococcus citri. Chromosoma 113:284-294

Buglia GL, Dionisi D, Ferraro M (2009) The amount of heterochromatic proteins in the egg is correlated with sex determination in Planococcus citri (Homoptera, Coccoidea). Chromosoma 118:737-746

Charnov EL (1982) The theory of sex allocation. Princeton University Press, Princeton

Charnov EL, Losdenhartogh RL, Jones WT, Van den Assem J (1981) Sex ratio evolution in a variable environment. Nature 289:27-33

Clark AB (1978) Sex ratio and local resource competition in a prosimian primate. Science 201:163-165

Cockburn A, Legge S, Double MC (2002) Sex ration in birds and mammals: can the hypotheses be disentangled? In: Hardy ICW (ed) Sex ratios: concepts and research methods. Cambridge University Press, Cambridge, UK 
R Development Core Team (2009) R: a language and environment for statistical computing. In: Team RDC (ed) R Foundation for Statistical Computing, Vienna, Austria

Gullan PJ, Kosztarab M (1997) Adaptations in scale insects. Annu Rev Entomol 42:23-50

Hadfield JD (2010) MCMC methods for multi-response generalized linear mixed models: the MCMCglmm R package. J Stat Softw 33:1-22

Hamilton WD (1967) Extraordinary sex ratios. Science 156:477-488

Hardy ICW (1992) Nonbinomial sex allocation and brood sex-ratio variances in the parasitoid hymenoptera. Oikos 65:143-158

James HC (1937) Sex ratios and the status of the male in Pseudococcinae (Hem. Coccidae). Bull Entomol Res 28:429-461

James HC (1938) The effect of the humidity of the environment on sex ratios from over-aged ova of Pseudococcus citri (Risso) (Hemipt. Coccidae). Proc R Entomol Soc Lond A 13:73-79

Kono M, Kogo R, Shimada M, Fukatsu T (2008) Infection dynamics of coexisting $\beta$ - and $\gamma$-proteobacteria in the nested endosymbiotic system of mealybugs. Appl Environ Microbiol 74:41754184

Murdoch WW, Nisbet RM, Luck RF, Godfray HCJ, Gurney WSC (1992) Size-selective sex-allocation and host feeding in a parasitoid host model. J Anim Ecol 61:533-541

Nelson-Rees WA (1960) A study of sex predetermination in the mealy bug Planococcus citri (Risso). J Exp Zool 144:111-137

Nur U (1980) Evolution of unusual chromosome systems in scale insects (Coccoidea: Homoptera). In: Blackman RL, Hewitt GM, Ashburner M (eds) Insect cytogenetics. Blackwell, Oxford, pp 97-118

Pen I, Weissing FJ (2002) Optimal sex allocation: steps towards a mechanistic theory. In: Hardy ICW (ed) Sex ratios: concepts and research methods. Cambridge University Press, London

Pinheiro J, Bates D, DebRoy S, Sarkar D, The R Core team (2007) nlme: linear and nonlinear mixed effects models. In: R package version 3.1-86. edn
Ross L, Langenhof MBW, Pen I, Beukeboom LW, West SA, Shuker DM (2010a) Sex allocation in a species with Paternal Genome Elimination: clarifying the role of crowding and female age in the mealybug Planococcus citri. Evol Ecol Res 12:89-104

Ross L, Pen I, Shuker DM (2010b) Genomic conflict in scale insects: the causes and consequences of bizarre genetic systems. Biol Rev 85(4):807-828

Roy M, Brodeur J, Cloutier C (2003) Temperature and sex allocation in a spider mite. Oecologia 135:322-326

Sabelis MW, Nagelkerke CJ, Breeuwer JAJ (2002) Sex ratio control in arrhenotokous and pseudo-arrhenotokous mites. In: Hardy ICW (ed) Sex ratios: concepts and research methods. Cambridge University Press, London

Schrader F (1921) The chromosomes of Pseudococcus nipae Biological Bulletin 40:259-270

Sheldon BC, West SA (2004) Maternal dominance, maternal condition, and offspring sex ratio in ungulate mammals. Am Nat 163:40-54

Trivers RL, Willard DE (1973) Natural selection of parental ability to vary sex-ratio of offspring. Science 179:90-92

Varndell NP, Godfray HCJ (1996) Facultative adjustment of the sex ratio in an insect (Planococcus citri, Pseudococcidae) with paternal genome loss. Evolution 50:2100-2105

Werren JH, Charnov EL (1978) Facultative sex-ratios and populationdynamics. Nature 272:349-350

West SA (2009) Sex allocation. Princeton University Press (Monographs in Population Biology Series), Princeton

West SA, Sheldon BC (2002) Constraints in the evolution of sex ratio adjustment. Science 295:1685-1688

West SA, Shuker DM, Sheldon BC (2005) Sex-ratio adjustment when relatives interact: a test of constraints on adaptation. Evolution 59:1211-1228

Wild G, West SA (2007) A sex allocation theory for vertebrates: combining local resource competition and condition-dependent allocation. Am Nat 170:E112-E128 